\title{
Optical design of six-mirror Anastigmat(6MA) off-axis freeform system
}

Baohua Wang, Wenchun Jiao, Ke Li, Yuanyuan Wang

Baohua Wang, Wenchun Jiao, Ke Li, Yuanyuan Wang, "Optical design of sixmirror Anastigmat(6MA) off-axis freeform system," Proc. SPIE 11852, International Conference on Space Optics - ICSO 2020, 1185208 (11 June 2021); doi: 10.1117/12.2599148

SPIE Event: International Conference on Space Optics - ICSO 2021, 2021, Online Only 


\section{International Conference on Space Optics-ICSO 2020}

Virtual Conference

30 March-2 April 2021

Edited by Bruno Cugny, Zoran Sodnik, and Nikos Karafolas
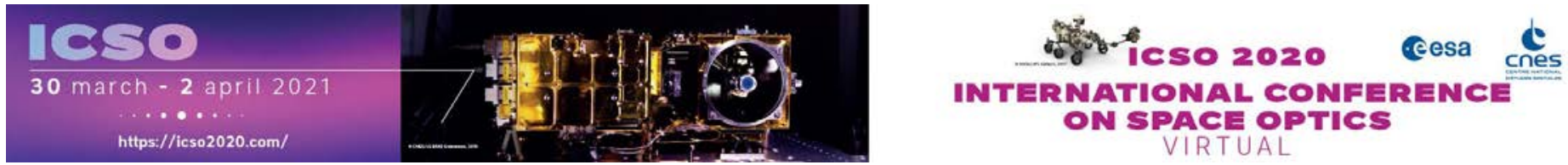

\section{Optical design of six-mirror Anastigmat(6MA) off-axis freeform system}

\section{Cesa isso procedings denes}




\title{
Optical Design of Off-axis Six-mirror Anastigmat (6MA) Freeform System with Wide Field of View and Low distortion
}

\author{
Baohua Wang, Wenchun Jiao, Ke Li, Yuanyuan Wang \\ Beijing Institute of Space Mechanics \& Electricity, Beijing 100094, China
}

\begin{abstract}
There is an urgent need for the imaging spectrometer with high spatial resolution and wide swathing range in the field of environment monitoring, agriculture evaluation and emergency disaster reduction. At the same time, higher request for the performance of spectrometers, such as signal-to-noise, is put forward to realize high-precision data inversion and quantitative detection. A telescope optical system with long focal length, large relative aperture and wide field-of-view is designed using for imaging spectrometer. The focal length is $1200 \mathrm{~mm}$, the relative aperture is $1 / 3$ and the field-of-view is $10^{\circ} \times 1^{\circ}$. A new re-imaging off-axis six-mirror anastigmat (6MA) configuration is adopted. It is beneficial to control the stray light and achieve the telecentric design in the image space. A real entrance pupil is located in front of the system to minimize the aperture of scanning mirror. The whole system is divided into two modules and each module can image perfectly at the axial field of view. Therefore, each module can be aligned and tested individually to reduce the alignment difficulty of the whole system. The off-axis freeform surface is used to control the distortion lower than $0.5 \%$ and advance the imaging quality. Finally, the manufacture and alignment tolerance are simulated and analyzed. The design and analysis results show that the six-mirror anastigmat (6MA) off-axis freeform system can satisfy the demand of high-performance hyperspectral imaging for earth monitoring. Good imaging quality and loose tolerances indicate that this optical system is of strong engineering application value.
\end{abstract}

Keywords: imaging spectrometer; 6MA; freeform surface; optical design

\section{INTRODUCTION}

Imaging spectrometer is a new type of optical remote sensing instrument which combines imaging technology with spectral technology. It can collect the spatial information, radiation information and spectral information of the target at the same time to form an integration data cube. It has important application value in the fields of atmosphere, land, ocean, agriculture and forestry, emergency disaster reduction and mineral resources investigation.

Typical spaceborne imaging spectrometers include Hyperion [1], COIS [2] and HyspIRI [3] et al. The spatial resolution of Hyperion and COIS is $30 \mathrm{~m}$, the spectral resolution is $10 \mathrm{~nm}$ and the swathing width is $7.5 \mathrm{~km}$ and $30 \mathrm{~km}$ respectively. The spatial resolution of HyspIRI is $60 \mathrm{~m}$, the spectral resolution is $10 \mathrm{~nm}$ and the swathing width is $150 \mathrm{~km}$. China has also developed the earth sensing imaging spectrometers carried on Tiangong-1[4], HJ-1A/B [5] and GF-5 satellites. The spatial resolution of the visible and shortwave infrared spectrometer on GF-5 satellite is $30 \mathrm{~m}$, the spectral resolution is $5 \mathrm{~nm}$ and $10 \mathrm{~nm}$ in the visible and shortwave infrared band respectively and the swathing width is over $60 \mathrm{~km} \mathrm{[6].}$

At present, the spectral resolution of spaceborne imaging spectrometers is generally $5 \mathrm{~nm} \sim 10 \mathrm{~nm}$, and the spatial resolution is ranged from $30 \mathrm{~m}$ to $60 \mathrm{~m}$. However, the swathing width is quite different, which the minimum swathing width is only $7.5 \mathrm{~km}$ and the maximum swathing width is $150 \mathrm{~km}$. Ecological environment monitoring, land resources and geological survey and other application fields often require high temporal resolution of remote sensor, which usually requires the swathing width could be greater than $100 \mathrm{~km}$. And the requirements of spatial resolution and spectral resolution are also higher and higher at the meantime. Current spectral data is hard to satisfy the application demand.

Therefore, in order to meet the urgent needs of high-performance imaging spectrometer, a telescope optical system with large field of view and large relative aperture is proposed in this paper. The working spectral range covers $0.4 \mu \mathrm{m} \sim 2.5 \mu \mathrm{m}$, the field of view is $10^{\circ} \times 1^{\circ}$ and the relative aperture is $1 / 3$. The spatial resolution can reach $14.1 \mathrm{~m}$ at the orbit of $705 \mathrm{~km}$, and the swathing width is over $120 \mathrm{~km}$. According to technical index requirement and imaging characters, the off-axis sixmirror optical system scheme is adopted. And the freeform surface is used to improve the imaging performance and reduce the distortion. Finally, the manufacture and alignment tolerances of the optical system are analyzed. 


\section{DESIGN REQUIREMENTS}

The spectrum of the telescope ranges from $0.4 \mu \mathrm{m}$ to $2.5 \mu \mathrm{m}$ and is divided into two imaging channels of $0.4 \mu \mathrm{m} \sim 1.0 \mu \mathrm{m}$ (VNIR) and $1.0 \mu \mathrm{m} \sim 2.5 \mu \mathrm{m}$ (SWIR). The detectors used in two channels are both the high frame rate devices. The pixel size of two detectors is $24 \mu \mathrm{m} \times 24 \mu \mathrm{m}$. The focal length of the optical system can be determined according to the pixel size, spatial resolution and orbit height. These parameters have the following relationship:

$$
f^{\prime}=\frac{d \times H}{G_{\mathrm{SD}}}
$$

Where $f^{\prime}$ is the focal length, $d$ is the pixel size, $H$ is the orbit height and $G_{\mathrm{SD}}$ is the ground space distance. When $d$ is equal $24 \mu \mathrm{m} 、 H$ is equal $705 \mathrm{~km}$ and $G_{\mathrm{SD}}$ is equal $14.1 \mathrm{~m}$, we can get the $f^{\prime}$ is equal $1200 \mathrm{~mm}$.

The field of view can be calculated according to the orbit height and swathing width:

$$
2 \omega=2 \arctan \left(\frac{I_{\mathrm{s}} / 2}{H}\right)
$$

Where $\omega$ is the half of the field of view, Is is the swathing width. When $I \mathrm{~s}$ is equal to $120 \mathrm{~km}$ and $H$ is equal to $705 \mathrm{~km}$, we can get the field of view is $9.73^{\circ}$ and take $10^{\circ}$ to design the optical system.

The relative aperture of the optical system has a great influence on the signal-to-noise ratio (SNR) and the optical modulation transfer function (MTF). And it also determines the volume and engineering difficulty of the optical system. By comprehensively analyzing the requirements of the visible, near-infrared and the shortwave infrared spectrum on the SNR and the MTF, the relative aperture of the optical system is selected as $1 / 3$. The MTF of diffraction limit is over $0.8 @ 20.81 \mathrm{p} / \mathrm{mm}$ in all spectral bands. The signal-to-noise ratio of VNIR spectrum is greater than 200, and the signal-tonoise ratio of the SWIR spectrum is greater than 100 .

In order to further improve the signal-to-noise ratio of the spectrometer, the scanning mirror is added in front of the system to compensate the integral time. The scanning angle is $\pm 7.3^{\circ}$ for the benefit of wider imaging area. The main technical parameters of the telescope optical system are summarized in Table 1.

Table.1 Technical parameters of optical system

\begin{tabular}{|c|c|}
\hline Technical parameters & Values \\
\hline Spectral band $/ \mu \mathrm{m}$ & $0.4 \sim 1.0 、 1.0 \sim 2.5$ \\
\hline Field of view/ & $\circ$ \\
\hline Focal length $/ \mathrm{mm}$ & $10 \times 1$ \\
\hline Relative aperture & 1200 \\
\hline Scanning angle $/{ }^{\circ}$ & $1 / 3$ \\
\hline
\end{tabular}

\section{DESIGN SCHEME AND ANALYSIS}

According to the technical parameters of the telescope optical system, several key issues should be considered in choosing optical system configuration and design process:

(1) Long imaging size

The optical system has long focal length and large field of view. So, the length of image plane is up to $210 \mathrm{~mm}$ and is much larger than the image length of the known imaging spectrometer currently in orbit and under development. The optical system structure with strong ability to suppress the off-axis aberration should be selected.

(2) High scanning accuracy

In order to improve the control accuracy of the scanning mirror, the aperture of the scanning mirror should be reduced as much as possible. When the scanning mirror is located near the entrance pupil of the system, it has the smallest aperture. Therefore, the optical system structure with the entrance pupil in front of the system should be considered.

(3) Good telecentricity in the image space

The spectral imaging system of the earth imaging spectrometer usually adopts the Offner structure. In order to match with the Offner spectral imaging system, the telescope optical system should have good telecentricity in the image space. 
(4) Lower straylight

Compared with panchromatic and multispectral imaging systems, stray light has a more serious impact on hyperspectral imaging systems. Therefore, the telescope optical system used in the imaging spectrometer should strictly control the straylight. The intermediate image plane is an important way to suppress the stray light of the optical system. Therefore, the re-imaging configuration with the intermediate image plane will be a better choice.

Considering the technical requirements and imaging characteristics of the optical system, the optical system in this paper adopts the design scheme as shown in Figure 1 below. The optical system has the intermediate image to suppress the stray light of the optical system effectively. The aperture stop is located in front of the primary mirror and is close to scanning mirror to reduce its size. The overall system is divided into two imaging modules by the intermediate image plane. The VNIR spectrum and SWIR spectrum share a set of optical system to realize the integrated design, and the two spectrums are separated through a certain field of view interval in Y direction to image respectively.

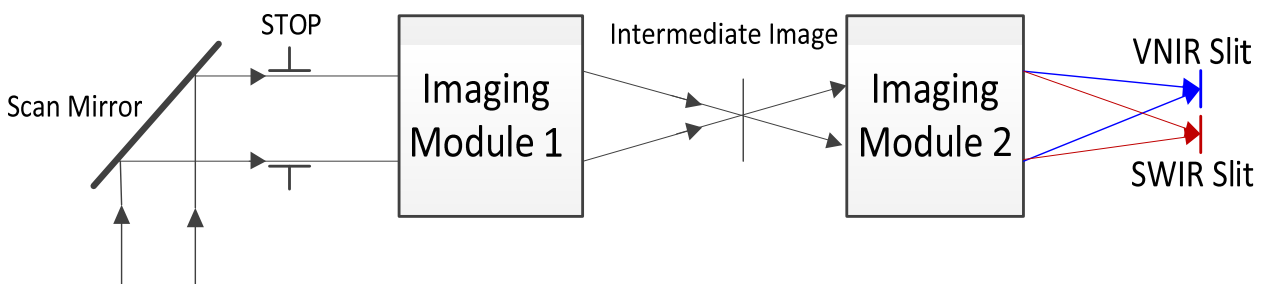

Fig.1 Scheme of optical system design

\section{OPTICAL SYSTEM DESIGN}

\subsection{Design result of the whole system}

According to the optical system design scheme, the optical system finally adopts a novel off-axis six-mirror configuration, which has good off-axis aberration correction ability and can realize the telecentric design. The whole optical system is divided into two imaging modules. Each imaging module contains three off-axis mirrors and the intermediate image plane is located between the two modules. The aperture stop is located in front of the primary mirror, and the scanning mirror is placed close to the aperture. After the design, the optical system layout is shown in Figure 2 below. The fourth mirror is a Zernike fringe polynomial freeform surface. Compared with spherical and aspheric surfaces, the freeform surface can advance the image performance, especially reduce the distortion.

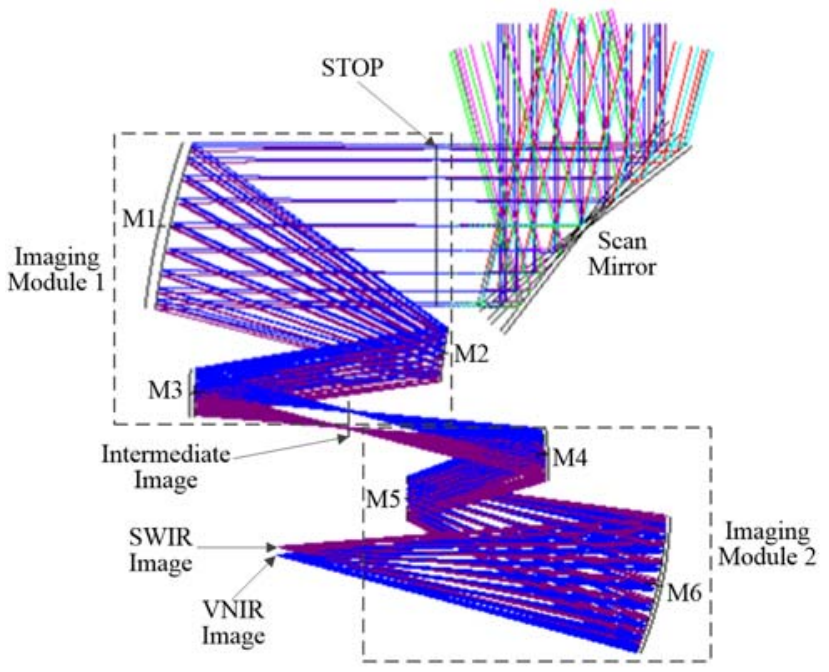

Fig.2 Schematic of off-axis six-mirror anastigmat (6MA) freeform system

The MTF curves of the whole optical system in VNIR and SWIR spectrums are shown in Figure 3 below. The minimum MTF value of different field of view is better than 0.87 and 0.76 in VNIR and SWIR spectrum respectively. 

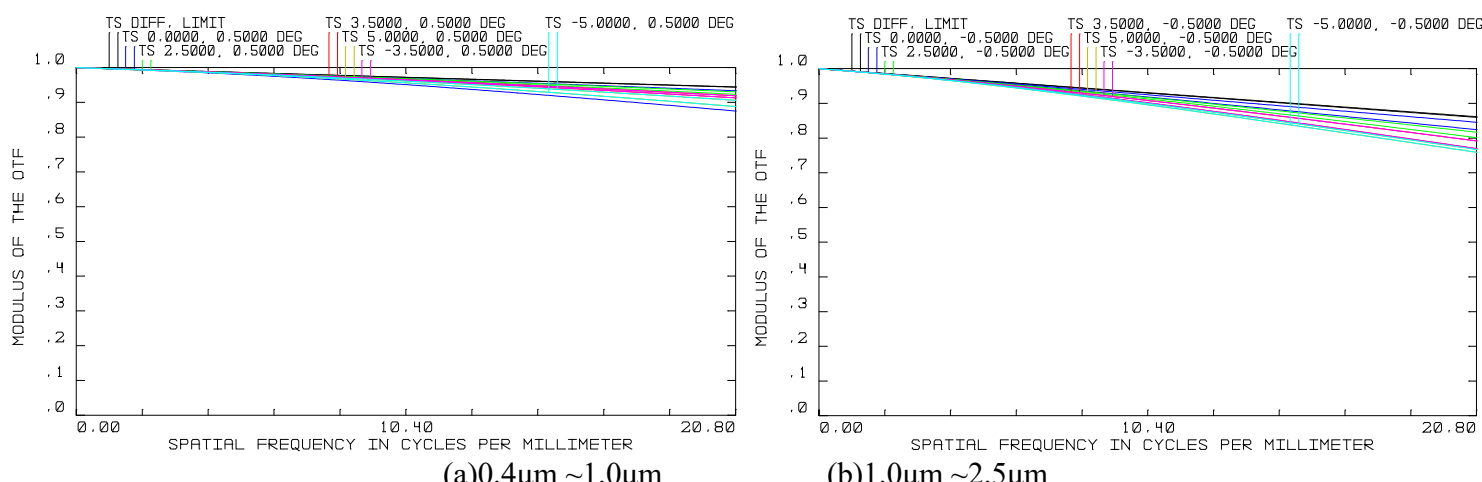

(b) $1.0 \mu \mathrm{m} \sim 2.5 \mu \mathrm{m}$

The spot diagram in VNIR and SWIR spectrums is shown in Figure 4. The RMS radius is small than $1 / 3$ pixel ( $8 \mu \mathrm{m})$, which can satisfy the imaging requirement.

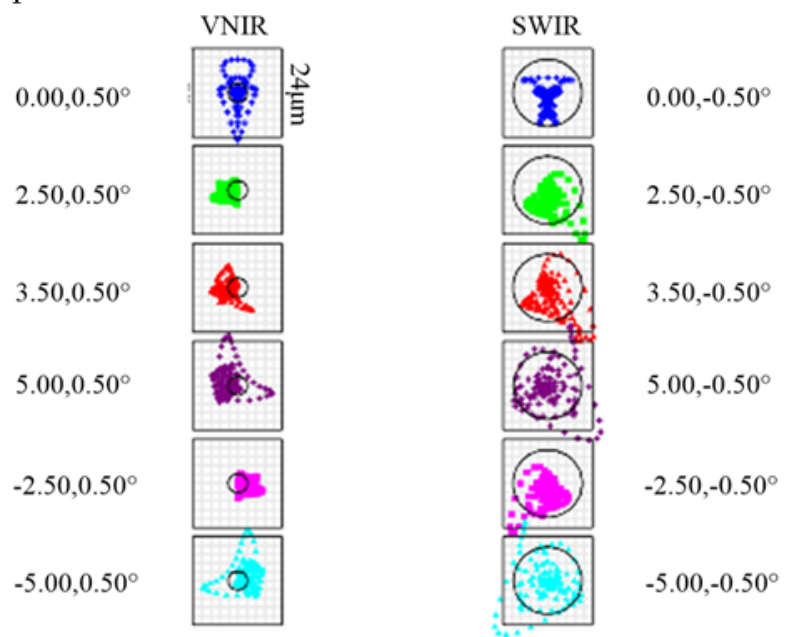

Fig.4 Spot diagrams of the whole optical system

The grid distortion is shown in Figure 5. The maximum relative distortion is below $0.4 \%$ within $10^{\circ} \times 1^{\circ}$ field of view. The low distortion is benefit for the remote sensing data processing and quick application.

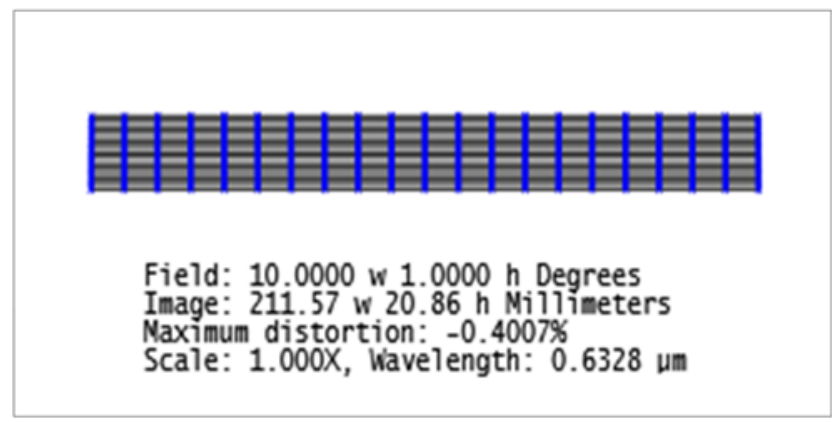

\subsection{Design result of each imaging module}

Fig.5 Grid distortion of the whole optical system

The more the number of optical elements there are, the greater the difficulty of the installation and adjustment of the optical system is. The off-axial system is more difficult than the coaxial system because the off-axial optical elements have more degree of freedom. In order to reduce the overall alignment difficulty of the off-axis six-mirror freeform system, not only the overall system can meet the design requirements, but also each of the two imaging modules has a certain degree of perfect imaging. Therefore, the assembly and test evaluation of the single imaging module can be carried out firstly, and 
then the two imaging modules can be combined to ensure the feasibility of the complex off-axis optical system and improve the alignment effect.

The independent optical system layout of imaging module is shown in Figure 6.

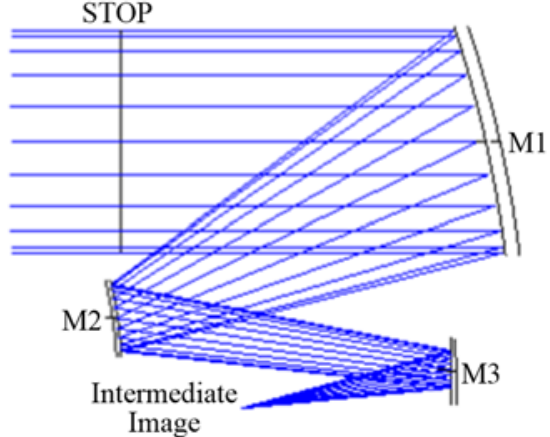

(a)Imaging module 1

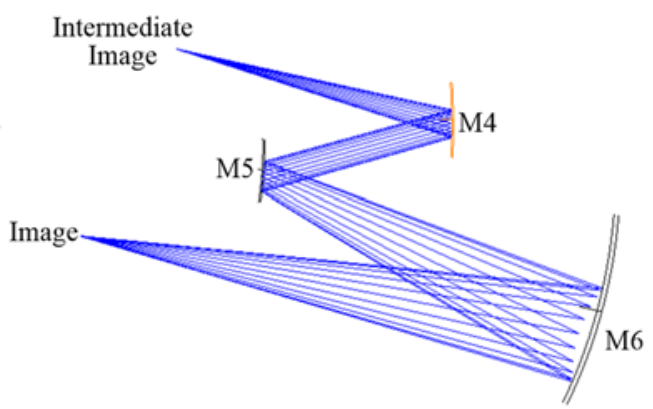

(b) Imaging module 2

Fig.6 Schematic of two optical system imaging modules

The MTF curves of the two imaging modules are shown in Figure 7 below. The MTF can reach the diffraction limit at the $0^{\circ}$ field of view, which benefit the alignment and evaluation.

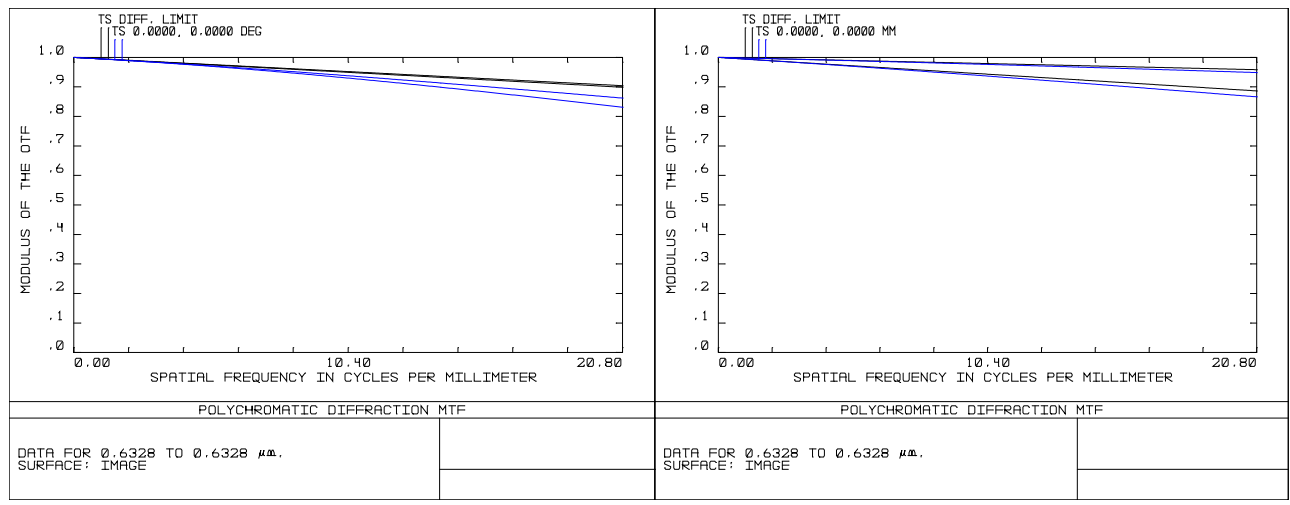

(a)Imaging Module1

(b) Imaging Module2

Fig.7 MTF curves of two optical system imaging modules

The wavefront map of the single imaging module is shown in Figure 8. The RMS wavefront error of the imaging module 1 is $0.11 \lambda(\lambda=0.6328 \mu \mathrm{m})$ and the RMS wavefront error of the imaging module 2 is $0.108 \lambda(\lambda=0.6328 \mu \mathrm{m})$, which can be evaluated by the interferometer.
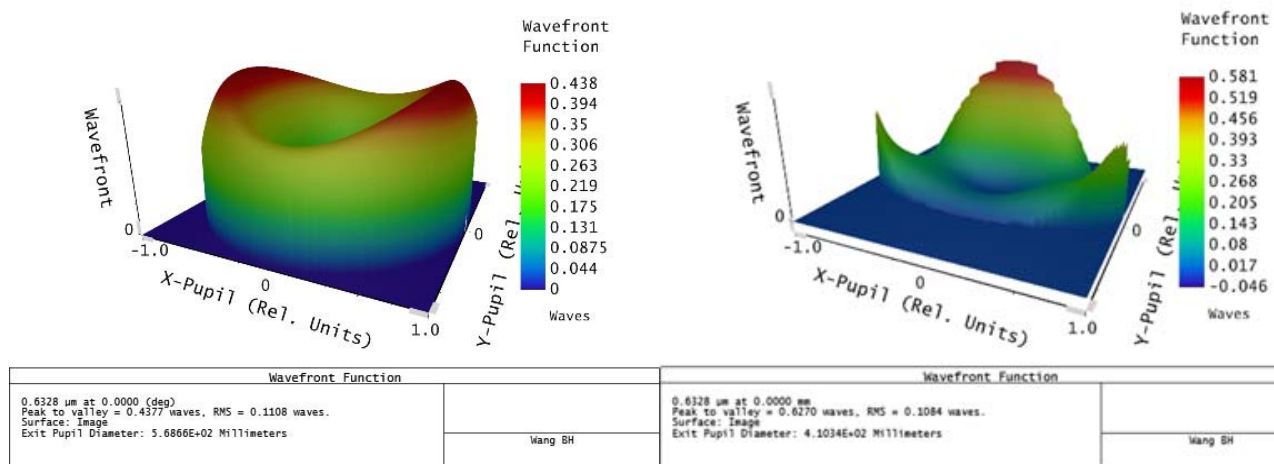

(a)Imaging module 1

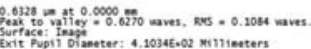

(b) Imaging module 2

Fig.8 Wavefront error of two optical system imaging modules 


\section{TOLERANCE ANALYSIS}

The optical system tolerances are analyzed to give the manufacture and alignment requirements. The back focal length and distance between two imaging modules are taken as the compensators. 500 Monte Carlo cycles are run and the statistics are given in Fig. 9. The average MTF values can be over 0.735 and 0.647 at the Nyquist frequency in the VNIR and SWIR spectrum respectively, which can also meet the specification.

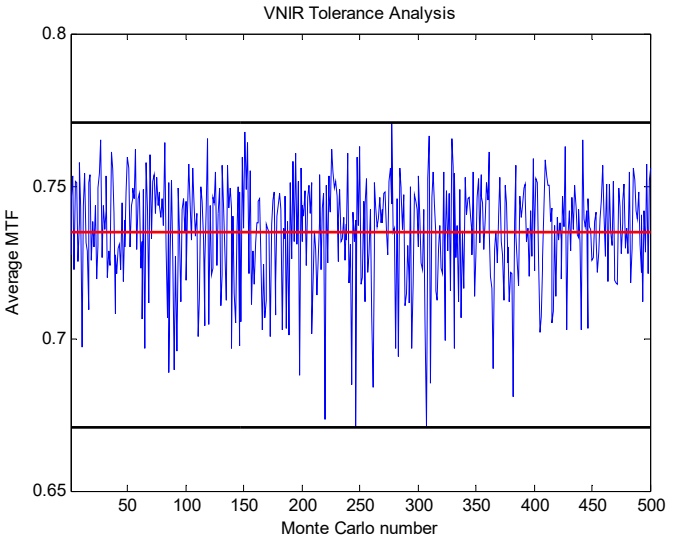

(a) $0.4 \mu \mathrm{m} \sim 1.0 \mu \mathrm{m}$

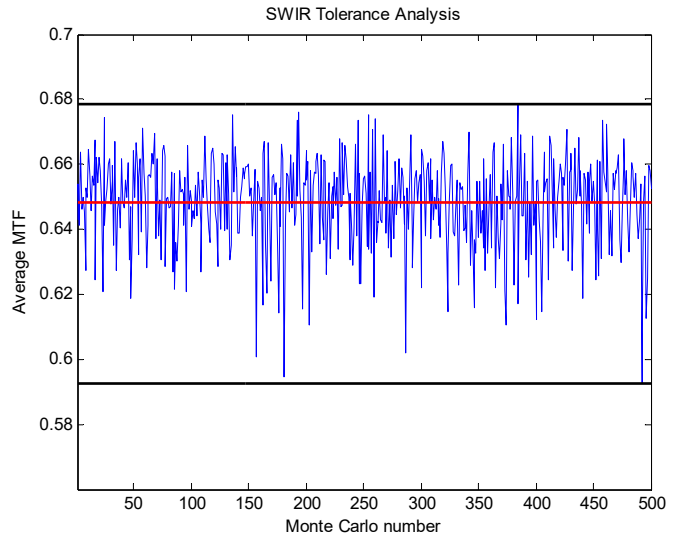

(b) $1.0 \mu \mathrm{m} \sim 2.5 \mu \mathrm{m}$

Fig.9 The tolerance analysis result of the whole optical system

\section{CONCLUSION}

Aiming at the deficiency of spatial resolution and swathing width of imaging spectrometer in orbit and under development and combining with the application requirements in different fields, a telescope optical system with large field of view and large relative aperture is designed. The working spectrum covers $0.4 \mu \mathrm{m} \sim 2.5 \mu \mathrm{m}$, the focal length is $1200 \mathrm{~mm}$, the relative aperture is $1 / 3$, and the field of view angle is $10^{\circ} \times 1.0^{\circ}$. The spatial resolution can achieve $14.1 \mathrm{~m}$ and swathing width is over $120 \mathrm{~km}$ at $705 \mathrm{~km}$ orbit altitude. A new type of off-axis six-mirror optical system configuration is proposed, which has the advantages of small scanning mirror size, high stray light suppression and good image telecentricity. The imaging quality of the whole optical system is close to the diffraction limit and each imaging module has a certain degree of perfect imaging. The alignment difficulty of the whole system is reduced through the modular assembly and test. The optical system tolerance of manufacture and alignment is loose, which is helpful to improve the engineering feasibility.

\section{REFERENCES}

[1] Pearlman J., Segal C., Lushalan L., et a1, "Development and operations of the EO-1 Hyperion imaging spectrometer", Proc. SPIE, 4135: 243 (2000).

[2] Curtiss O. Davis, Donald Horan, Mike Corson, "On-Orbit calibration of the naval earthmap observer (NEMO) coastal ocean imaging spectrometer", Proc. SPIE, 4132(2000).

[3] Christine M. Lee, Morgan L, Cable, Simon J, et a1, "An introduction to the NASA Hyperspectral Infrared Imager (HyspIRI) mission and preparatory activities", Remote Sensing of Environment, 2167:6-19 (2015).

[4] Wang Y. M., Jia J. X., He Z. P., et al, "Key technologies of advanced hyperspectral imaging system”, Journal of Remote Sensing, 1007-4619: 0850-08 (2016).

[5] Xiang L. B., Wang Z. H., Liu X. B., et a1, "Spatially modulated Fourier transform hyperspectral imager for HJ1A satellite", Spacecraft Engineering, 18(6): 43-49 (2009).

[6] Liu Y. N., "Visible-shortwave Infrared Hyperspectral Imager of GF-5 Satellite", Spacecraft Recovery \& Remote Sensing, 39(3):25-28 (2018). 